\title{
On-Line Independent Tap-Changing of Each Feeder Supplied by a Low Voltage Distribution Transformer
}

\author{
David Johnston \\ Faculty of Engineering and Environment, Northumbria University, Newcastle upon Tyne, UK \\ Email: david.johnston@northumbria.ac.uk
}

Received December 2013

\begin{abstract}
An on-line tap-changing circuit was developed for use with low voltage transformers $(10 \mathrm{kV} / 380 \mathrm{~V}$, or equivalent), in which the tap positions could be set independently for each low voltage feeder. This allows for possible variation in loads and distributed generation between different feeders fed from a given transformer, allowing the line voltages to be kept within limits on all feeders. A combination of computer simulation and practical experiments was used. A model constructed in Excel gave preliminary results, which was used to specify a more detailed model in Matlab ${ }^{\circledR}$ Simulink. A small-scale $220 / 380 \mathrm{~V}$ distribution network was constructed, with currents limited to $5 \mathrm{~A}$ per phase. Finally, a rotary switch was constructed, suitable for currents up to $500 \mathrm{~A}$, which would be required for a full-scale low voltage distribution network. The results showed that the voltage could be kept within limits, even with a large difference in load and distributed generation from one feeder to another.
\end{abstract}

\section{Keywords}

Tap-Changing; On-Line; Feeders; Independent

\section{Introduction}

Electricity suppliers are required to maintain line voltages within certain limits centered about the nominal voltage. (This is $-6 \% /+10 \%$ in the EU, and $\pm 10 \%$ is used in many other countries around the world [1] [2].) To enable this under changing loading conditions, transformers have multiple tap settings, allowing the output voltage to be varied in such a way that the supply voltage remains within limits along the full length of each feeder [3]. Medium voltage transformers (33/10 kV, or equivalent) have on-line tap changing, allowing them to respond in real time to changing loading conditions. However, this means that all the $10 \mathrm{kV}$ feeders from a given transformer, and all the $380 \mathrm{~V}$ feeders fed from them, change their voltages as a single unit, with no independent control from one feeder to another. Low voltage transformers $(10 \mathrm{kV} / 380 \mathrm{~V}$, or equivalent) have off-line tap changing. This does not provide real time control; rather it anticipates future loading, until such loading changes, at which time the taps must be reset. However, it does provide a limited degree of independent control between different low voltage networks. 
Increasingly, low power distributed generation is being installed on the distribution network. This includes renewable energy sources (photovoltaic (PV), wind, etc) and combined heat and power (CHP) generators [4]. Utility-owned generators will be installed on the $380 \mathrm{~V}, 10 \mathrm{kV}, 35 \mathrm{kV}$ (or higher) sections-depending on output power, and will be controlled by the network operator [5] [6]. Similar generators may be installed by private supply companies, and arrangements may be put in place for these to be controlled by the network operators. Generators used by property owner/occupiers and installed on their premises-on-site generation-will largely be small-scale, and installed on the $380 \mathrm{~V}$ network [7]. (A typical exception would be a large generator installed on industrial premises.) These will be operated by the building occupiers according to their needs and priorities, and even where an agreement exists with the network operator, the degree of external control will generally be limited. These generators will reduce the currents in the lines, thus reducing the voltage drop produced by loads, and in extreme cases they will reverse the current flow, resulting in a voltage rise along the line. In the case of on-site generation, this will not generally be subjected to network control.

Provision of on-line tap changing on $10 \mathrm{kV} / 380 \mathrm{~V}$ transformers will allow independent real-time control of different $380 \mathrm{~V}$ networks fed from a given $10 \mathrm{kV}$ feeder. This will allow the line voltages to be maintained within limits for a wider range of loading generating conditions. However, this will not provide independent control of the feeders supplied by a given $10 \mathrm{kV} / 380 \mathrm{~V}$ transformer, which would be required where the loading and/or generation varied greatly between feeders. A situation where this might occur is as follows. One or more feeders supply domestic premises, which have a significant quantity of installed PV systems. Another feeder supplies small commercial premises (e.g., shops), which have little or no installed generation. During the working day, the domestic loads are light, and PV generation is high, resulting in excess generation and voltage rises along these feeders. At the same time, the commercial loads are high, resulting in a voltage drop along the relevant feeder. It may not always be possible to maintain the voltages within limits for all the feeders, with all of them connected to the same tap of the transformer.

By placing the taps on the output side of the transformer, it is possible for each feeder to be connected to a different tap. The line voltage along each feeder can then be kept within limits by measuring the line voltage at the far end of the feeder (and at other points, if necessary) and changing the tap setting accordingly. This can increase the range of loading/generation conditions which can be accommodated on a given low voltage network.

\section{Computer Simulation}

An Excel based modelling tool and a Matlab ${ }^{\circledR}$ Simulink were used to simulate a low voltage network. The Excel modelling tool allowed a range of conditions to be modelled in a short time, and a selection of these were used as the basis for modelling in Simulink.

\subsection{Excel Based Model}

The Excel modelling tool used a simple resistive network to model a set of low voltage feeders. Each feeder is divided into sections, with the length and cable type specified by the user, thus determining the resistance of each section. Loads and distributed generators are located at nodes between sections, with the power of each specified by the user. The difference between these is the net power. The resistance of each (net) load is calculated based on the rated voltage $\left(R=V^{2} / P\right)$. Thus, generators are represented as negative resistances. (Modelling these as nonlinear sources in combination with resistive loads and lines would require iterative solutions.) This results in the voltages being approximate. However, the modeling tool allows the network parameters (loads, line resistances, etc.) to be changed quickly, so that a wide range of operating conditions can be investigated in a short time. An appropriate selection of these can then be analysed further in Simulink. The first and second series of models were for a fixed tap, and a common on-line tap for all feeders respectively. The results for a common on-line tap are shown in Figure 1. As seen, the voltage on the feeder with distributed generation exceeds the maximum limit, while the voltage for the heavily loaded feeder is below the minimum limit. Therefore, there is no common tap setting, for which the voltages on all the lines will be within the limits. By changing the tap settings for each feeder separately, it is possible to keep the voltages on both feeders within limits along their entire length, as shown in Figure 2.

\subsection{Simulink Model}

A Simulink model was used for more detailed and accurate analysis of the scenarios selected using the Excel 


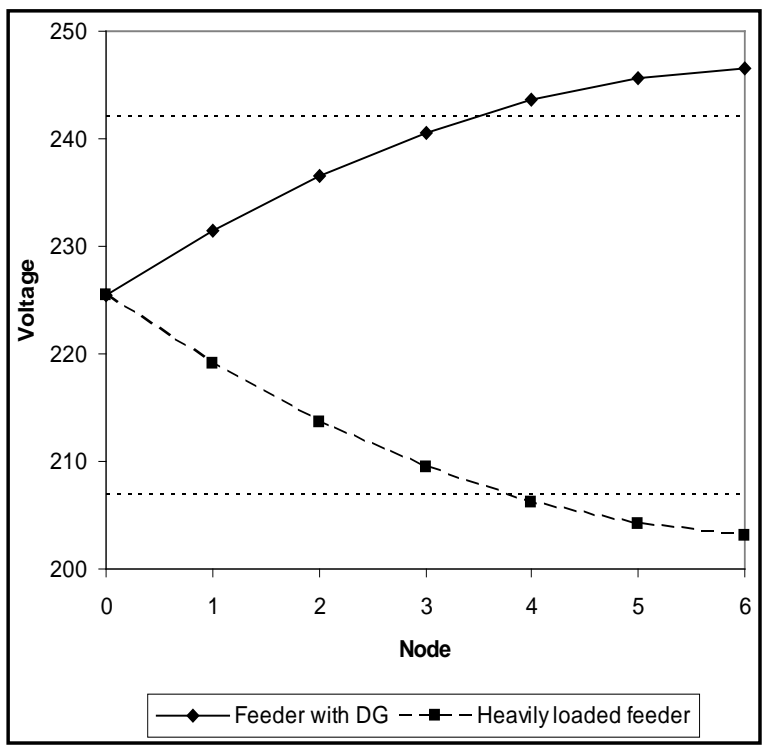

Figure 1. Line voltages along feeders connected to a common tap $(102.5 \%=225.5 \mathrm{~V})$.

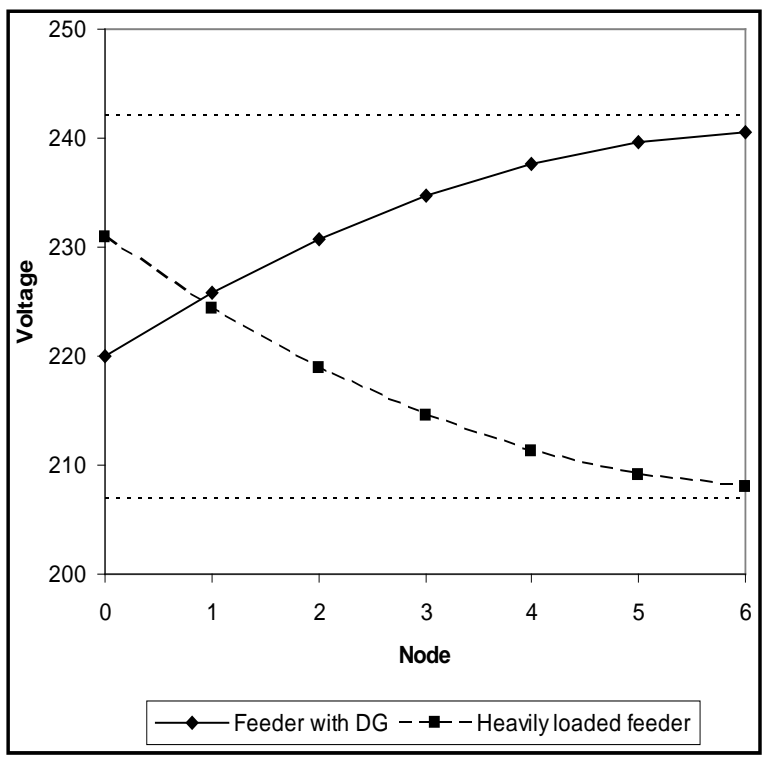

Figure 2. Line voltages along feeders connected to separate taps $(100 \%=220 \mathrm{~V} ; 105 \%=231 \mathrm{~V})$.

modeling tool. A circuit diagram of the model is shown in Figure 3. For clarity, a single feeder and a single phase are shown, and the feeder is reduced to two line sections and two nodal loads. These are shown here as resistances, but in general resistive/inductive elements are used. The tap-changing transformer is represented by two transformers (identified as "Main" and "Tap"), with their inputs connected in parallel and the outputs connected in series. The main transformer has outputs at $0 \mathrm{~V}$ and the minimum of the tapping range. The tap transformer has five outputs covering the tapping range from minimum to maximum voltage. Each of these tap outputs is connected to a contactor, which is connected to the near end of the feeder. A control block closes one of these contactors, connecting the feeder to the required tap. The control block receives a voltage measurement signal from the far end of the feeder. If the far-end voltage is below the minimum limit, the control block increases the tap setting by one step. If the far-end voltage is above the maximum limit, the control block decreases the tap setting by one step. 


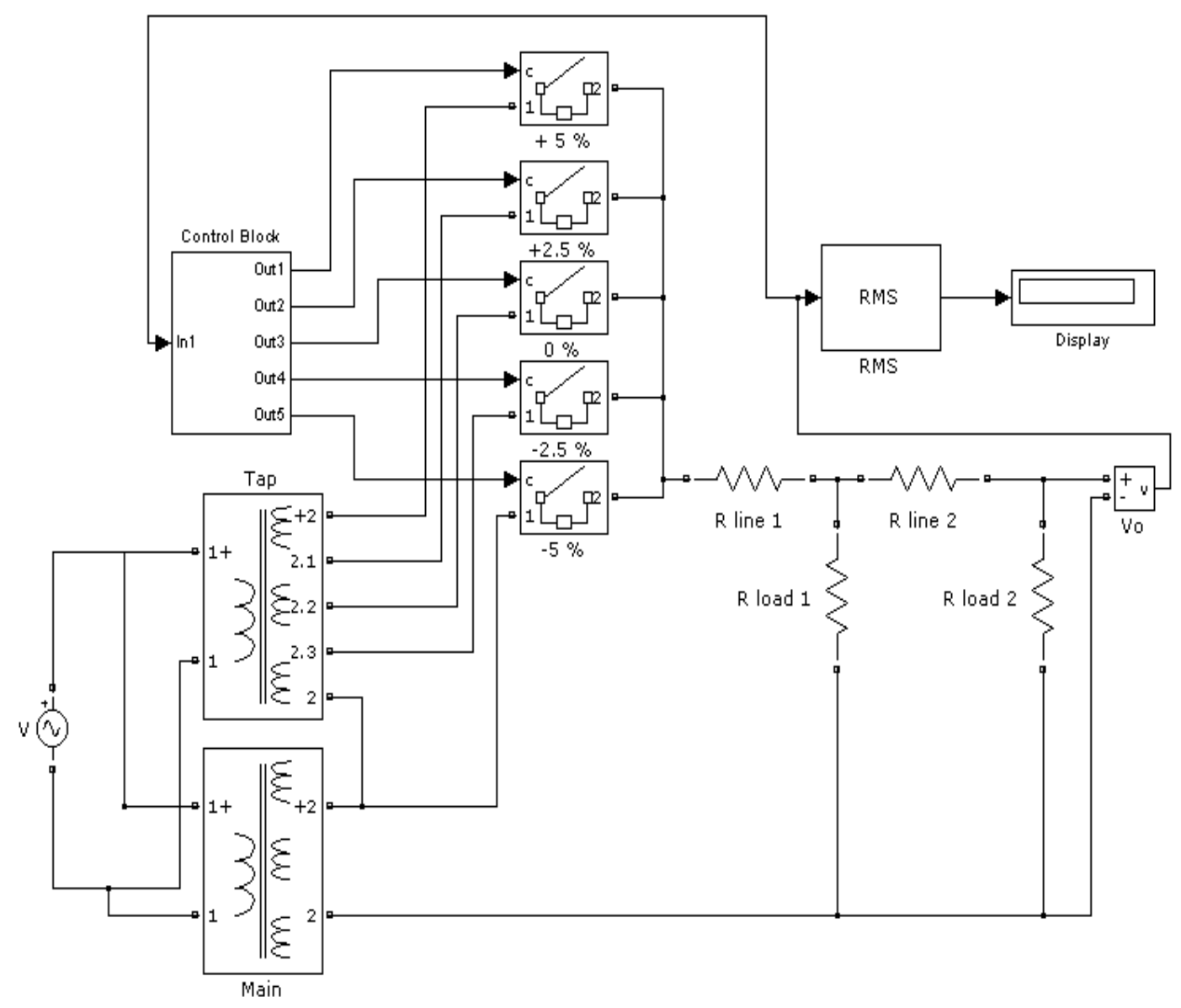

Figure 3. Simulink model of OLTC transformer and a feeder (one phase).

A number of scenarios were tested, each with a large loading/generation differential between feeders. In one example, $300 \mathrm{~kW}$ of net loads were place on the nodes along one feeder, and up to $240 \mathrm{~kW}$ of net generation on each of the remaining feeders. With independent tap setting, the far-end voltage on the heavily loaded feeder was $208 \mathrm{~V}$, and the far-end voltage on the feeder with the largest level of distributed generation was $240 \mathrm{~V}$. Both of these voltages are within the limits.

\section{Practical Evaluation}

Two experimental arrangements were used, which were based on the limitations of the power available in the laboratory—a reduced version of a transformer/feeder network, and a rotary switch capable of passing the currents typical of a low voltage network.

\subsection{Reduced Power Low Voltage Network}

This network was a practical implementation based on the Simulink model. An Elgar Electronics Smart Wave ${ }^{\mathrm{TM}}$ power generator was used to supply the Chinese standard voltage of 220/380 V from the (United Kingdom) 230/400 V supply. The current limit of this generator is 5 A per phase, and this set the limit for the rest of the equipment/circuits used.

The main transformer was made up from two toroidal transformers with dual $55 \mathrm{~V}$ outputs. These were connected in series to give $220 \mathrm{~V}$ (nominal), which was reduced to $210 \mathrm{~V}$ when supplied at $220 \mathrm{~V}$. The tap transformer was a multi-tap transformer with outputs of 0 - 6 - 12 - 18 - $24 \mathrm{~V}$ (nominal), giving a range of $23 \mathrm{~V}$ when supplied by $220 \mathrm{~V}$. By connecting this in series with the toroidal transformers, a tapping range from 210 to 233 $\mathrm{V}$ was available. This arrangement was replicated for each phase. 
The contactors were implemented by three-pole relays, which simultaneously changed the tap settings for all three phases of a given feeder. A block of five such relays was connected to each feeder. The output stage of the control block was a digital decoder, thus ensuring that only one relay in each block was closed at any given time, to avoid shorting of the outputs of the multi-tap transformer.

Because of the 5 A per phase limit, the resistances of the loads were higher than those of a real network. Load resistances of $220 \Omega$ to $2.2 \mathrm{k} \Omega$ were used, giving a maximum of $1 \mathrm{~A}$ at each node. (Not all nodes drew this maximum current.) In order to produce voltage drops similar to those on real feeders, in the presence of smaller currents, larger line resistances were used. The total resistance per phase of each feeder was up to $20 \Omega$, made up of sections of 1 to $4 \Omega$. The resistors used have inductances giving $X / R$ ratios similar to those for real cables and loads. A single generator (driven by a D.C. motor) was used to represent the larger number of generators that would generally be present on a real system.

Figure 4 shows the results for a lightly loaded feeder with a $200 \mathrm{~W}$ generator at the far end, and a heavily loaded feeder. The results are in general agreement with those of the Simulink model, and the voltages on both feeders are within limits.

\subsection{High Current Rotary Switch}

A rotary switch was constructed with a current rating typical of currents in a low voltage network. On-line tap changers used at higher voltages use break-before-make contacts, in order to avoid shorting between taps. However, the resultant interruption of current can cause high voltage transients, where inductive components are present in the system. One solution is to place resistive elements between the contacts of the tap changer, such that they only come into circuit during a transition between tap settings. This method was used in the design of the rotary switch. A cross section is shown in Figure 5. A cam, embedded in the rotor, connects to one of the contacts placed around the rim, thus connecting to the corresponding transformer tap. This cam is connected along the axis to a slip ring, which is connected via a contact to the relevant feeder. The cam and contacts are made of phosphor bronze to reduce friction. Graphite sections are placed either side of the rotary cam. During a tap transition, as the rotor is rotating, one of the graphite sections makes contact with the next contact, thus providing a continuous conductive path. This avoids voltage transients due to inductive components, while also providing sufficient resistance to avoid shorting of adjacent taps. A stepper motor was used to rotate the rotor, in response to overvoltage or undervoltage signals.

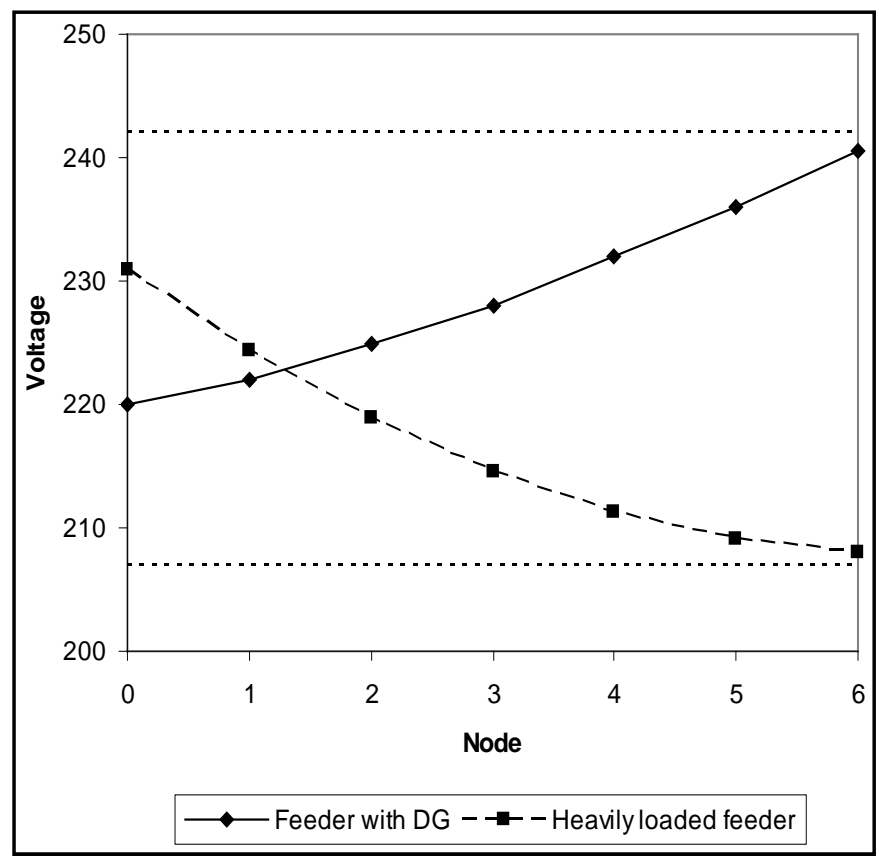

Figure 4. Line voltages for a low-power network with a single generator on one feeder. 


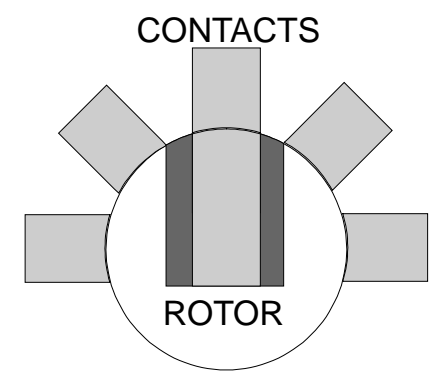

Figure 5. Cross section of the rotary switch, showing cam and contacts.

A 10 V, 300 A D.C. power supply was used to evaluate the conductivity of the switch. Temperature measurements showed that there was a moderate increase above ambient, but not sufficient to cause overheating. In order to test the performance during switching, a $230 \mathrm{~V}$ A.C. supply and an inductive load of $2 \mathrm{mH}$. Resistive heating of the graphite was negligible and voltage transients were less than $150 \%$ of the steady-state voltage.

\section{Conclusions}

The tap-changing system was tested under a range of conditions, focusing on those where there is a large loading/generation differential between feeders. Independent tap setting was able to keep the voltages on all feeders within limits, under operating conditions in which a fixed or common tap would result in voltages exceeding the limits. This allows a greater range of loads and particularly distributed generation to be installed. It may also reduce or avoid the need for alternative network support measures, such as battery storage. Electric vehicles (EVs) have the potential to act as such storage, but in high density residential areas, home charging may not be possible, and EVs would not be available for network support. In such cases, independent tap changing could provide the degree of support necessary to allow the use of in-situ generation. Transformers for independent tap setting would require multiple taps on the secondary coils. The cost involved could effectively be reduced by installing such coils in new transformers, to replace older units, as part of the maintenance/upgrade of the low voltage networks.

The analysis in this work considered loads and generators distributed along each feeder, which resulted in voltages falling or rising steadily along each feeder. However, if a large load or generator is placed part-way along a feeder, the resulting minimum or maximum voltage would be located at this point. An additional voltage measurement would be needed at this point, to ensure that the voltage is within limits along the entire length of the feeder. The control system could be designed to allow additional voltage measurement inputs after installation, to allow for large loads or generators being installed on the network at a later time.

\section{Acknowledgements}

The author would like to thank Edward Bentley, Tianxiang Jiang and Pasist Suwanapingcarl, whose previous research contributed to this work.

\section{References}

[1] EN 50160_European Committee for Electrotechnical Standardisation.

[2] GB/T 12325 (2003) Admissible Deviation of Supply Voltage.

[3] Weedy, B.M. (1994) Electric Power Systems. John Wiley \& Sons.

[4] Aly, G.E.M., El-Zeftawy, A.A., El-Hefnawy, A.A. and Eraky, S.A. (1999) Technical Assessment of Electric UtilityInteractive Residential Photovoltaic Systems. Electric Power Systems Research, 51, 175-185. http://dx.doi.org/10.1016/S0378-7796(98)00159-X

[5] Milanović, J.V. (2001) Characteristics of Voltage Sags in Radial Networks with Dynamic Loads and Embedded Generators. Proceedings of the IEEE Power Technology Conference, Porto.

[6] Ackermann, T., Andersson, G. and Söder, L. (2001) Distributed Generation: A Definition. Electric Power Systems Re- 
search, 57, 95-204. http://dx.doi.org/10.1016/S0378-7796(01)00101-8

[7] El-Khattam, W. and Salama, M.M.A. (2004) Distributed Generation Technologies, Definitions and Benefits. Electric Power Systems Research, 71, 119-128. http://dx.doi.org/10.1016/j.epsr.2004.01.006 